\title{
GYNÄKOLOGIE IM NATIONALSOZIALISMUS: DIE GEBURTSHILFLICH-GYNÄKOLOGISCHE KLINIK DES LANDESKRANKENHAUSES (LKH) GRAZ 1938-1945
}

Am Institut für Sozialmedizin und Epidemiologie der Medizinischen Universität Graz läuft derzeit unter der Leitung von ao. Univ. Prof. Èva Rásky ein vom FWF finanziertes Projekt zur Geschichte der Universitätsfrauenklinik Graz während des Nationalsozialismus. Es gehört zu einer Reihe von wissenschaftlichen Aktivitäten, die von der 1998 an der Medizinischen Fakultät Graz gegründeten Arbeitsgruppe „Die Rolle der Medizin im Nationalsozialismus in der Steiermark“ initiiert wurden.

Seit dem „Anschluss“ Österreichs an das Deutsche Reich waren auch die österreichischen Gynäkologen in die nationalsozialistische Bevölkerungs- und Rassenpolitik involviert. Nicht nur die nationalsozialistische Machtergreifung an der Klinik und ihre personalpolitischen Konsequenzen, wie die Ersetzung des politisch missliebigen Klinikvorstands Hans Zacherl durch den „reichsdeutschen“ SS-Führer Karl Ehrhardt, sondern auch die zahlreichen Zwangssterilisationen und Schwangerschaftsabbrüche - so eine Hypothese - dürften Klinik und Forschung radikal verändert haben. Für viele Patientinnen war die Klinik kein Ort der Hilfe mehr, sondern ein Ort der Gefahr für Leib und Leben. Sie waren nicht nur ärztlichen Körpereingriffen gegen ihren Willen ausgesetzt, sondern wurden auch missbräuchlich medizinischen Versuchen unterzogen. Sterilisandinnen, schwangere Zwangsarbeiterinnen und offenbar auch Sloweninnen hatten unter diesen Übergriffen am meisten zu leiden, doch auch an „erbgesunden“ „deutschen“ Patientinnen wurde experimentiert.

Ziel des Projekts ist es, die Geschichte der Grazer Geburtshilfe und Gynäkologie im Nationalsozialismus als eine Geschichte des Arzt-Patientinnen-Verhältnisses zu schreiben, in der medizinhistorische, sozialhistorische und zeitgeschichtliche Perspektiven verknüpft werden sollen. Die als Sozialgeschichte der Gynäkologie im Nationalsozialismus konzipierte Studie wird durch die Synopse folgender Untersuchungsfelder entstehen:

1. die Analyse der wissenschaftlichen Produktion der Grazer Gynäkologen und deren Verortung im Diskurs der zeitgenössischen scien-

tific community, 
2. die Rekonstruktion der politischen, wissenschaftlichen und sozialen Netzwerke der in der geburtshilflich-gynäkologischen Klinik tätigen Ärzte und Ärztinnen,

3. die Rekonstruktion der Patientinnenstruktur durch Auswertung überlieferter Klinikakten und die Erhebung sozialgeschichtlicher Daten ausgewählter Patientinnen.

Erste Ergebnisse sind nachzulesen. ${ }^{1}$

1 Vgl. Gabriele CZARNOWSKI, Vom „reichen Material ... einer wissenschaftlichen Arbeitsstätte“. Zum Problem missbräuchlicher medizinischer Praktiken an der Grazer Universitäts-Frauenklinik in der Zeit des Nationalsozialismus, in: Wolfgang FREIDL, Werner SAUER (Hg.), NS-Wissenschaft als Vernichtungsinstrument. Rassenhygiene, Zwangssterilisation, Menschenversuche und NS-Euthanasie in der Steiermark (Wien 2004) 225-273. 